\title{
Local Measures of Entanglement and Critical Exponents at Quantum Phase Transitions
}

\author{
L. Campos Venuti, ${ }^{1,2}$ C. Degli Esposti Boschi, ${ }^{1,3}$ G. Morandi, ${ }^{1,2,3}$ M. Roncaglia,,${ }^{1,2,3}$ and A. Scaramucci ${ }^{1}$ \\ ${ }^{1}$ Dipartimento di Fisica, V.le C. Berti-Pichat 6/2, I-40127 Bologna, Italy \\ ${ }^{2}$ INFN Sezione di Bologna, V.le C. Berti-Pichat 6/2, I-40127 Bologna, Italy \\ ${ }^{3}$ CNR-INFM, Unità di Bologna, V.le C. Berti-Pichat 6/2, I-40127 Bologna, Italy
}

(Dated: 13th November 2018)

\begin{abstract}
We discuss on general grounds some local indicators of entanglement, that have been proposed recently for the study and classification of quantum phase transitions. In particular, we focus on the capability of entanglement in detecting quantum critical points and related exponents. We show that the singularities observed in all local measures of entanglement are a consequence of the scaling hypothesis. In particular, as every non-trivial local observable is expected to be singular at criticality, we single out the most relevant one (in the renormalization group sense) as the best-suited for finite-size scaling analysis. The proposed method is checked on a couple of one-dimensional spin systems. The present analysis shows that the singular behaviour of local measures of entanglement is fully encompassed in the usual statistical mechanics framework.
\end{abstract}

PACS numbers: 05.70.Jk, 03.75.Ud, 75.10.Pq

The entanglement properties of condensed matter systems have been recently object of intensive studies [1, 2], especially close to quantum critical points (QCP) where quantum fluctuations extend over all length scales. Moreover, the amount of entanglement in quantum states is a valuable resource that promotes spin systems as candidates for quantum information devices [3, 4].

Since the seminal studies on the interplay between entanglement and quantum critical fluctuations in spin $1 / 2$ models [1, 5], several works suggested different local measures of entanglement (LME) as new tools to locate QCP's [6, 7, 8, 9, 10, 11, 12]. The term local here is meant for measures which depend on observables that are local in real space. This has to be contrasted with global measures of entanglement, e.g. the block entropy [2, 13], or the so-called localizable entanglement [14, 15], which are aimed to capture the entanglement involved in many degrees of freedom.

The picture that has emerged so far seems to be non systematic and model-dependent. Some of the (local) indicators reach the maximum value at QCP's [9], while others show a singularity in their derivatives [1, 5, 6, 12]. Close to the transition, the system being more and more correlated, one expects naïvely an increase of entanglement. However it seems that the maxima observed in single-site entropies have to be ascribed to a symmetry of the lattice Hamiltonian that does not necessarily correspond to a QCP. For example, in the 1D Hubbard model the single-site entropy reaches the maximum possible value at $U=0[9]$. This is due to the equipartition of the empty, singly-, and doubly-occupied sites rather than to the Berezinski-Kosterlitz-Thouless (BKT) transition occurring at that point. In fact, in the anisotropic spin-1 system discussed below, the equipartition points do not coincide with the transition lines which are not marked by any symmetry of the lattice model [16].

The onset of non-analyticity in two commonly used entanglement indicators (concurrence and negativity) was recently proved in [17] for models with two-body interactions. Let us first argue, from a statistical mechanics point of view, that this result is in fact more general: as a consequence of the scaling hypothesis every local average displays a singularity at the transition with the exception of accidental cancellations. In particular as any LME is built upon a given reduced density matrix, the former will inherit the singularities of the entries of the latter.

A second order quantum phase transition is characterized by long-ranged correlation functions and a diverging correlation length $\xi$. Let the transition be driven by a parameter $g$ such that the Hamiltonian is

$$
\mathcal{H}(g)=\mathcal{H}_{0}+g \mathcal{V}
$$

At $T=0$ the free energy density reduces to the ground state energy density which shows a singularity in the second (or higher) derivatives with respect to $g$ :

$$
\frac{1}{L}\langle\mathcal{H}(g)\rangle=e(g)=e_{\mathrm{reg}}(g)+e_{\text {sing }}(\xi(g)),
$$

where $\xi \approx\left|g-g_{c}\right|^{-\nu}$ is the correlation length, $g_{c}$ is the critical point and $L$ is the number of sites. Note that, as a consequence of the scaling hypothesis, the singular part of the energy $e_{\text {sing }}$ is a universal quantity that depends only on $\xi$, the relevant length scale close to the critical point. Hence, $e_{\text {sing }}$ may be considered quite in general an even function of $g-g_{c}$ around the critical point.

Differentiating $e(g)$ with respect to $g$, gives the mean value $\langle\mathcal{V}\rangle / L$, whose singular part $\mathcal{O}_{g}$ behaves as

$$
\mathcal{O}_{g} \approx \operatorname{sgn}\left(g-g_{c}\right)\left|g-g_{c}\right|^{\rho} .
$$

Scaling and dimensional arguments imply that $\rho=$ $(d+\zeta) \nu-1$ where $d$ is the spatial dimensionality and $\zeta$ is the dynamical exponent. For the sake of clarity here we set $\zeta=1$ as occurs in most cases [18]. For a second order phase 
transition $\rho>0$. In particular, if $0<\rho \leq 1$ the next derivative will show a divergence [25]

$$
\mathcal{C}_{g} \equiv \frac{\partial^{2} e}{\partial g^{2}} \approx\left|g-g_{c}\right|^{\rho-1} .
$$

In the case where $g$ is mapped to the temperature $T$ in the related $(d+1)$-statistical model, $\mathcal{C}_{g}$ will correspond to the specific heat and $\rho=1-\alpha$ (Josephson's scaling law). As far as entanglement is concerned, the singular term $\mathcal{O}_{g}$ appears in every reduced density matrix containing at least the sites connected by the operator $\mathcal{V}$. Obviously, modulo accidental cancellations, any function (i.e. entanglement measures) depending on such density matrix, displays a singularity with an exponent related to $\rho$. The renormalization group theory allows us to be even more general: to the extent that a local operator can be expanded in terms of the scaling operators (permitted by the symmetries of the Hamiltonian), its average will show a singularity controlled by the scaling dimension of the most relevant term.

From an operational point of view, LME's have been employed mainly to detect the transition point using finite-size data. Following the previous discussion we can argue that, in a typical situation, the best suited operator for a finite-size scaling (FSS) analysis is precisely $\langle\mathcal{V}\rangle$ for the following reasons. First because it naturally contains the most relevant operator, whose average $\mathcal{O}_{g}$ has the smallest possible critical exponent $\rho$. Second the occurrence of $\operatorname{sgn}\left(g-g_{c}\right)$ in Eq. (1) plays an important role in finding the critical point, in case its location is not known from analytical arguments. The FSS theory asserts [19] that in a system of length $L$,

$$
\mathcal{O}_{g}(L) \approx \operatorname{sgn}\left(g-g_{c}\right) L^{-\rho / \nu} \Phi_{\mathcal{O}}(L / \xi),
$$

where $\Phi_{\mathcal{O}}(z)$ is a universal function which must behave as $z^{\rho / \nu}$ in order to recover Eq. (1) in the (off-critical) thermodynamic limit $L \gg \xi$. In the critical regime $z \rightarrow 0, \Phi_{\mathcal{O}}(z)$ must vanish in order to avoid jump discontinuities for finite $L$. Notice that the sign of the microscopic driving parameter $\left(g-g_{c}\right)$ survives in the FSS for $\mathcal{O}_{g}$. As a consequence, since $\mathcal{O}_{g}(L)$ is an odd function of $\left(g-g_{c}\right)$, the curves $\mathcal{O}_{g}(L)$ at two successive values of $L$ as a function of $g$ cross at a single point $g_{L}^{*}$ near $g_{c}$ (see below). In this way, by extrapolating the sequence $g_{L}^{*}$ to $L \rightarrow \infty$ one has a useful method for detecting numerically the critical point. Surprisingly, to our knowledge the present method was not considered in the past, in favor of the so-called phenomenological renormalization group (PRG) method [19]. However the PRG method exploits the scaling of the finite-size gap which requires the additional calculation of an excited level typically computed with less accuracy than the ground state. This means that the computational time is roughly doubled. Another advantage w.r.t. the PRG method is that we do not have the complication of two crossing points $g_{L}^{*}$. In fact as $\Delta(g) \sim \xi^{-1} \sim\left|g-g_{c}\right|^{\nu}$ is an even function, the scaled gaps will cross at two values of $g$ for each $L$.

Once $g_{c}$ is determined, using FSS techniques the critical exponents $\rho$ and $\nu$ may be extracted simply by estimating $\rho / \nu=d+1-1 / \nu$. In order to find other possible critical exponents, we should perturb our model with other operators permitted by the symmetries $\mathcal{H} \rightarrow \mathcal{H}+g^{\prime} \mathcal{V}^{\prime}$ and repeat the same study near $g^{\prime}=0$.

In what follows we will illustrate these ideas in two different $d=1$ spin models: $i$ ) the thoroughly studied, exactly solvable Ising model in transverse field for which all arguments can be checked analytically and ii) the spin- $1 X X Z$ Heisenberg chain with single-ion anisotropy for which there are no analytical methods to locate the different transition lines.

Ising Model in Transverse Field. We consider the following Hamiltonian with periodic boundary conditions (PBC)

$$
H=-\sum_{i=1}^{L}\left[\sigma_{i}^{x} \sigma_{i+1}^{x}+h \sigma_{i}^{z}\right],
$$

where the $\sigma^{\alpha}$ 's are the Pauli matrices. This model exhibits a QCP at $h=1$, where it belongs to same universality class as the 2D classical Ising model, with central charge $c=1 / 2$. From the exact solution, it is possible to show that the transverse magnetization $m^{z}=\left\langle\sigma_{i}^{z}\right\rangle$, obtained differentiating the energy w.r.t. the driving parameter $h$, has the following expression near the transition point $h=1$

$$
m^{z} \simeq \frac{2}{\pi}-\frac{h-1}{\pi}(\ln |h-1|+1-\ln 8) .
$$

As expected, $m^{z}$ is a continuous function at the transition point $h=1$, showing a singular part which is manifestly odd in $h-1$. The next $h$-derivative exhibits a logarithmic divergence, as it is related to the specific heat in the corresponding 2D classical model. Most important for us is the "crossing effect" near the critical point of the family of curves $m^{z}(L)$, for different $L$. In Fig. $1 m^{z}(L)$ is plotted for several system sizes. It is evident that, increasing $L$, the crossing points converge rapidly to $h=1$.

A quantitative analysis of the crossing effect may be done in the spirit of FSS, considering separately the critical regime ( $L \ll \xi)$ and the off-critical one $(L \gg \xi)$ for any finite $L$. In the off-critical regime, the finiteness of the correlation length $\xi$, reflects in the exponential convergence of the energy to the thermodynamic value

$$
e_{L}(h)=e_{\infty}(h)+\frac{\left|h^{2}-1\right|^{1 / 2}}{\sqrt{\pi}} \frac{e^{-L / \xi}}{L^{3 / 2}}\left[1+O\left(L^{-1}\right)\right],
$$

with $\xi$ given by the formula $\sinh (1 / 2 \xi)=|1-h||h|^{-1 / 2} / 2$, from which we can read the critical exponent $\nu=1$, for $h \rightarrow$ 1 . In the critical regime, the finite-size expression for the transverse magnetization is

$m_{L}^{z}(h) \simeq \frac{2}{\pi}+\frac{\ln (L)+\ln (8 / \pi)+\gamma_{C}-1}{\pi}(h-1)+\frac{\pi}{12} \frac{1}{L^{2}}$,

where $\gamma_{C}=0.5772 \ldots$ is the Euler-Mascheroni constant. The finite-size critical field $h_{c, L}$ is obtained via the crossing points between curves with slightly different lengths, 


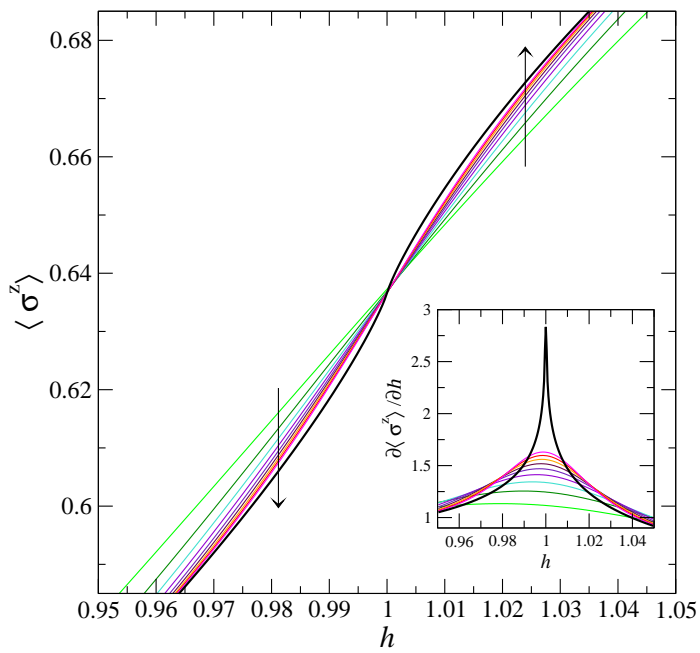

Figure 1: The transverse magnetization $\left\langle\sigma_{i}^{z}\right\rangle$, is plotted versus $h$ for various sizes $L$ ranging from 20 to 100 in steps of 10 . The black thick line corresponds to the thermodynamic limit. The arrows indicate the direction of increasing $L$. The inset shows the derivative w.r.t. $h$.

$$
\begin{aligned}
m_{L}^{z}\left(h_{c, L}\right)= & m_{L+2}^{z}\left(h_{c, L}\right) . \text { The solution is } \\
& h_{c, L}=1+\frac{\pi^{2}}{6} \frac{1}{L^{2}}+O\left(L^{-3}\right),
\end{aligned}
$$

showing a convergence towards the critical point as fast as $L^{-2}$.

As we stressed already, the singularities of local averages reflect in the behavior of LME's. Among these, the simplest measures the entanglement between one site and the rest of the system and is given by the von Neumann entropy $S_{1}=-\operatorname{Tr} \rho_{1} \ln \rho_{1}$, where $\rho_{1}$ is the reduced single-site density matrix. For spin- $1 / 2$ systems $\rho_{1}$ is simply written in terms of Pauli matrices

$$
\rho_{1}=\frac{1}{2}\left(\mathbb{I}+m_{x} \sigma^{x}+m_{y} \sigma^{y}+m_{z} \sigma^{z}\right) .
$$

For the Ising model (3) $m_{y}=m_{x}=0$ and the single site entropy behaves as $S_{1} \sim-0.239(h-1) \ln |h-1|$ so that its $h$-derivative diverges logarithmically. A non-zero value of $m^{x}$ is possible if spontaneous symmetry breaking is taken into account, by adding a small longitudinal (i.e. along $x$ ) field that tends to zero after the thermodynamic limit is performed. In this case $\sigma_{i}^{x}$ becomes the most relevant operator and $m_{x}=\theta(1-h)\left(1-h^{2}\right)^{1 / 8}$. Accordingly the singular part of the entropy is $S_{1} \sim(1-h)^{1 / 4}$ for $h<1$. The same singularities are encountered in all the single-site measures built upon $\left(\rho_{1}\right)^{2}$, e.g. purity and linear entropy [6].

On the same line one can consider LME's based on the two-site density matrix $\rho_{i j}$, obtained taking the partial trace over all sites except $i$ and $j$. The entries of $\rho_{i j}$ now depend also on the two-point correlation functions $\left\langle\sigma_{i}^{\alpha} \sigma_{j}^{\beta}\right\rangle$. In accordance with the general theory, all such averages behave as $(h-1) \ln |h-1|$ close to the critical point. In the case of nearest-neighbour sites this explains the logarithmic divergence in the first derivative of the concurrence $C(1)$, as found in [5]. Instead the leading singularity in the next-nearest neighbour concurrence $C(2)=$ $\left[\left\langle\sigma_{i}^{x} \sigma_{i+2}^{x}\right\rangle-\left\langle\sigma_{i}^{y} \sigma_{i+2}^{y}\right\rangle+\left\langle\sigma_{i}^{z} \sigma_{i+2}^{z}\right\rangle-1\right] / 2$ turns out to be of the form $(h-1)^{2} \ln |h-1|[5]$. This is due to the accidental cancellation of the $(h-1) \ln |h-1|$ terms contained in the correlators.

Spin-1 Heisenberg Chain with Anisotropies. Let us now consider the non-integrable spin-1 model

$$
H=\sum_{i}\left[S_{i}^{x} S_{i+1}^{x}+S_{i}^{y} S_{i+1}^{y}+\lambda S_{i}^{z} S_{i+1}^{z}+D\left(S_{i}^{z}\right)^{2}\right]
$$

which shows a rich phase diagram [16]. It is known that the transition line between the large- $D$ phase (where the spins tend to lie in the $x y$-plane) and the Haldane phase (characterized by non zero string order parameters) is described by a conformal field theory with central charge $c=1$ [20]. This means that the critical exponents change continuously along the critical line. For the detection of the $c=1$ critical line, the PRG [21] or the twisted-boundary method [16] have been used in the literature. We have tested the finite-size crossing method outlined above, fixing $\lambda=2.59$ for which previous studies ensure $\rho<1$ [20]. The driving parameter being now $D$, the quantity to consider is $\partial e / \partial D$ which, by translational invariance reduces to $\left\langle\left(S_{i}^{z}\right)^{2}\right\rangle \equiv \mathcal{O}_{D}$. In Fig. 2] we plot $\mathcal{O}_{D}$ versus $D$ for various sizes $L$. The crossing points of the curves for subsequent values of $L$, determined by $\mathcal{O}_{D}(D, L)=\mathcal{O}_{D}(D, L+10)$, converge rapidly to the critical point $D_{c}=2.294$ consistently with the phase diagram reported in [16].

The effective theory in the continuum limit of the model (7) around the $c=1$ line reduces to the sine-Gordon Hamiltonian density [20]

$$
\mathcal{H}_{S G}=\frac{1}{2}\left[\Pi^{2}+\left(\partial_{x} \Phi\right)^{2}\right]-\frac{\mu}{a^{2}} \cos (\sqrt{4 \pi K} \Phi) .
$$

The coefficient $\mu$ is zero along the critical line, $a$ is a short distance cut-off of the order of the lattice spacing and $K$ is related to the compactification radius, varying continuously between $1 / 2$ and 2 along the critical line. In this framework, crossing the critical line in the lattice model (7) means going from negative to positive values of $\mu$ and the corresponding $\mu$-derivative gives $\mathcal{O}_{\mu}=\langle\cos (\sqrt{4 \pi K} \Phi)\rangle$. From the sineGordon theory [23] it is known that $\mathcal{O}_{\mu} \approx \operatorname{sgn}(\mu)|\mu|^{K /(2-K)}$ and $\xi \approx|\mu|^{1 /(K-2)}$. In our case $\mu \approx\left(D-D_{c}\right)$ at fixed $\lambda$, so that $\rho=K /(2-K), \nu=1 /(2-K)$. On the one hand, the critical exponent $\rho / \nu=K$, can be independently calculated from the conformal spectrum obtained numerically, as explained in Ref. [20] giving $K=0.76$. On the other hand, from the FSS of the derivatives of $\mathcal{O}_{D}$ at $D=D_{c}$ (shown in the inset of Fig.2 we find $K=0.78$ showing that the method presented here is effective for the calculation of the critical 


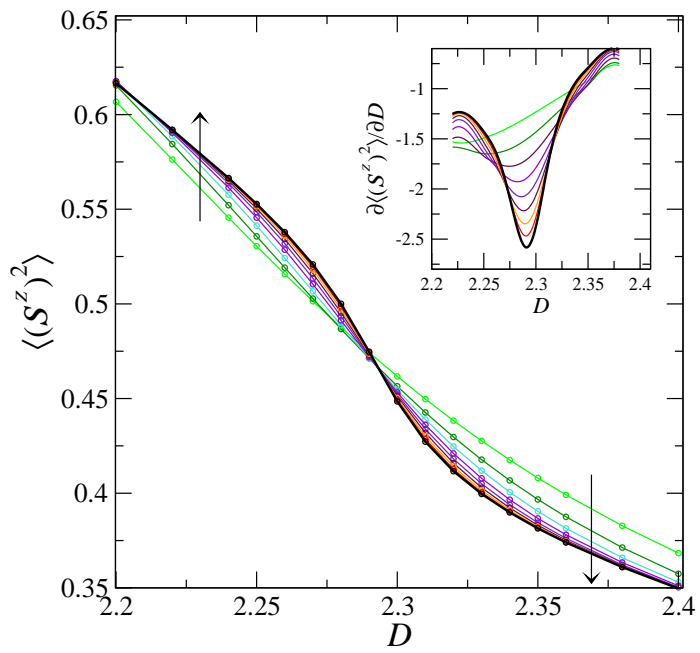

Figure 2: The single-site average $\mathcal{O}_{D}=\left\langle\left(S_{i}^{z}\right)^{2}\right\rangle$, is plotted versus $D$ for various sizes $L$ ranging from 20 to 100 (thick line) in steps of 10 . The arrows indicate the direction of increasing $L$ and the inset shows numerical derivative w.r.t. $D$, interpolated with splines. The data have been obtained via a DMRG program [22] using 400 optimized states and 3 finite system iterations with PBC

exponent as well. Since the same transition can be driven by $\lambda$ at fixed $D$, we checked that sitting at $D=2.294$ we obtained $\lambda_{c}=2.591$ by looking at $\mathcal{O}_{\lambda}=\left\langle S_{i}^{z} S_{i+1}^{z}\right\rangle$. According to our general discussion, a similar behavior is seen also in $\mathcal{O}_{D}$ even if it is a single-site indicator.

The scaling exponent $\rho / \nu$ in Eq. (2) is best obtained from the analysis of the first derivative when $\rho<1$. As we move towards the BKT point, $K \rightarrow 2, \nu \rightarrow \infty$, so the divergence should be seeked in derivatives with increasing order. Accordingly the crossing method (as well as the PRG) becomes less efficient as we approach the BKT point, for which a finer analysis is needed involving level spectroscopy [24].

Again the singularities of local averages enter the LME's. In the spin-1 case, thanks to the symmetries of the Hamiltonian (7) the the single-site entropy reads

$$
S_{1}=-\mathcal{O}_{D} \log \left(\frac{\mathcal{O}_{D}}{2}\right)-\left(1-\mathcal{O}_{D}\right) \log \left(1-\mathcal{O}_{D}\right),
$$

where $0<\mathcal{O}_{D}<1$ in any bounded region of the phase diagram. Note that the maximum of $S_{1}$ occurs for $\mathcal{O}_{D}=2 / 3$, which is not related to any phase transition, but simply signals the equipartition between the three states $|+1\rangle,|0\rangle,|-1\rangle$. This occurs for example at the isotropic point $(\lambda=1, D=0)$ where the system is known to be gapped. Similarly, in the Ising model $S_{1}$ is maximal at $h=0$, i.e. when $m_{z}=0$, where no transition occurs. Therefore the intuitive idea of the local entropy $S_{1}$ being maximal as a criterion to find quantum phase transitions [9], seems to be more related to symmetry arguments rather than to criticality.

In this Letter we have put in evidence the origin of singularities in LME's which have been recently proposed to detect QCP's. Typically, apart from accidental cancellations, such singularities can be traced back to the behavior of the transition-driving term $\mathcal{V}$ and to the corresponding scaling dimension. Moreover the FSS of $\langle\mathcal{V}\rangle$ turns out to be a valuable method to determine the critical point and the associated exponents. This method has been illustrated for a couple of spin models displaying qualitatively different QCP's. More generally, these considerations can be directly transposed to other many-body problems, like strongly interacting fermionic systems. Our arguments indicate that the singular behavior of LME's can be adequately understood in terms of statisticalmechanics concepts. Physically, the understanding of the intimate relation between genuine multipartite entanglement and the critical state remains an open challenge. From this perspective, it may be useful to conceive nonlocal indicators that could unveil the role of non-classical correlations near criticality.

We are grateful to E. Ercolessi, F. Ortolani and S. Pasini for useful discussions. This work was supported by the TMR network EUCLID (No. HPRN-CT-2002-00325), and the COFIN projects 2002024522_001 and 2003029498_013.

[1] T. J. Osborne and M. A. Nielsen, Phys. Rev. A 66, 32110 (2002).

[2] G. Vidal, J. I. Latorre, E. Rico, and A. Kitaev, Phys. Rev. Lett. 90, 227902 (2003).

[3] S. Lloyd, Science 261, 1569 (1993).

[4] C. H. Bennett and D. P. DiVincenzo, Nature (London) 404, 247 (2000).

[5] A. Osterloh, L. Amico, G. Falci, and R. Fazio, Nature (London) 416, 608 (2002).

[6] R. Somma, G. Ortiz, H. Barnum, E. Knill, and L. Viola, Phys. Rev. A 70, 042311 (2004).

[7] J. Vidal, G. Palacios, and R. Mosseri, Phys. Rev. A 69, 022107 (2004).

[8] T. Roscilde, P. Verrucchi, A. Fubini, S. Haas, and V. Tognetti, Phys. Rev. Lett. 93, 167203 (2004).

[9] S.-J. Gu, S.-S. Deng, Y.-Q. Li, and H.-Q. Lin, Phys. Rev. Lett. 93, 86402 (2004).

[10] P. Zanardi, Phys. Rev. A 65, 042101 (2002).

[11] M.-F. Yang, Phys. Rev. A 71, 030302(R) (2005).

[12] A. Anfossi, P. Giorda, A. Montorsi, and F. Traversa, Phys. Rev. Lett. 95, 056402 (2005).

[13] P. Calabrese and J. Cardy, JSTAT p. P06002 (2004).

[14] F. Verstraete, M. A. Martín-Delgado, and J. I. Cirac, Phys. Rev. Lett. 92, 087201 (2004).

[15] L. Campos Venuti and M. Roncaglia, Phys. Rev. Lett. 94, 207207 (2005).

[16] W. Chen, K. Hida, and B. C. Sanctuary, Phys. Rev. B 67, 104401 (2003).

[17] L.-A. Wu, M. S. Sarandy, and D. A. Lidar, Phys. Rev. Lett. 93, 250404 (2004).

[18] S. Sachdev, Quantum Phase Transitions (Cambridge, 1999).

[19] M. N. Barber, in Phase Transitions and Critical Phenomena, edited by C. Domb and J. L. Lebowitz (Academic Press, 1983), vol. 8.

[20] C. Degli Esposti Boschi, E. Ercolessi, F. Ortolani, and M. Roncaglia, Eur. Phys. J. B 35, 465 (2003).

[21] U. Glaus and T. Schneider, Phys. Rev. B 30, 215 (1984). 
[22] S. R. White, Phys. Rev. B 48, 10345 (1993).

[23] S. Lukyanov and A. Zamolodchikov, Nucl. Phys. B 493, 571 (1997).

[24] A. Kitazawa, K. Nomura, and K. Okamoto, Phys. Rev. Lett. 76,
4038 (1996).

[25] In general, care must be taken to account for logarithmic singularities see i.e. [19] 